\title{
Iidemyia, um novo gênero neotropical de Pachygastrinae (Diptera, Stratiomyidae)
}

\author{
José Roberto Pujol-Luz ${ }^{1}$
}

${ }^{1}$ Departamento de Zoologia, Instituto de Ciências Biológicas, Universidade de Brasília, Campus Darcy Ribeiro, Asa Norte, $70910-900$ Brasília-DF, Brasil. jrpujol@unb.br

\begin{abstract}
Iidemyia, a new neotropical genus of Pachygastrinae (Diptera, Stratiomyidae). Iidemyia, gen.nov., is proposed for Panacris microdonta Kertész (new record from Brazil, States of Espírito Santo and São Paulo). The new genus differs from the allied genera Panacris, Spyridopa, and Tijucameru in the male terminalia, shape of the scutellum and scutellar spines, and the presence of a supra-alar callus.
\end{abstract}

KEYWORDS. Biodiversity; Brazil; taxonomy.

RESUMO. Iidemyia, um novo gênero neotropical de Pachygastrinae (Diptera, Stratiomyidae). Iidemyia, gen.nov., é proposto para Panacris microdonta Kertész (novo registro no Brasil, Estados do Espírito Santo e São Paulo). O novo gênero difere dos gêneros afins Panacris, Spyridopa e Tijucameru na terminália do macho, forma do escutelo e espinhos escutelares, e pela presença do calo supra-alar.

PALAVRAS-CHAVE. Biodiversidade; Brasil; taxonomia.

Os Pachygastrinae constituem um grupo heterogêneo de Stratiomyidae com grandes variações principalmente no tamanho e na coloração, bem como na forma das antenas, na venação alar, ornamentações e número de espinhos escutelares. Na Região Neotropical ocorrem 56 gêneros, destes 34 são monotípicos e 27 ocorrem no Brasil, onde existem 62 espécies conhecidas (modificado de Woodley 2001). Nas poucas revisões feitas para táxons dessa subfamília é reduzido o número de chaves para identificação dos gêneros, tanto para a fauna mundial (Lindner 1964) quanto para as faunas regionais (James 1967, James et al.1980).

James et al. (1980) fizeram a revisão de 22 gêneros de Pachygastrinae, com a descrição de novos táxons. No gênero Panacris, descreveram duas novas espécies e expressaram dúvidas a respeito da permanência de $P$. microdonta nesse gênero, principalmente por causa da forma, posição e do número de espinhos escutelares, e da presença do calo póssutural supra-alar, uma peculiar projeção mesotorácica em forma de concha sobre os escleritos alares de ambas as asas ("tegula" de James et al. 1980; e "Praealarcallus" de Kertész 1908). Os autores caracterizaram $P$. microdonta da seguinte maneira: "Easily recognizable from all other know species in the genus [Panacris] by the presence of a small but clearly evident, flattened lobe-like extension of mesonotum, somewhat in the form of a projecting tegula (Praealarcallus of Kértész) just above each wing base, and by the unusually small spines of the scutellum. The scutellum is semi-oval, broadly rounded apically, with a pair of small spines, each about one-fourth to one-fifth the length to the scutellum ... ", e seguem: "In other species of the genus the scutellum is trapezoidal in form and transverse apically, its spines much larger and set on its apical corners". Após outros comentários afirmam que esta é uma das espécies de Panacris que deveria ser transferida para outro gênero.

O gênero Panacris Gerstacker, 1857 é representado atualmente por sete espécies neotropicais, das quais quatro ocorrem no Brasil: P. breviseta Lindner, 1964, P. lucida Gerstacker, 1857, P. microdonta Kertész, 1908 e P. nigribasis Lindner, 1964 (modificado de Woodley 2001); destas, apenas P. lucida e P. microdonta estão representadas em coleções no Brasil.

Pujol-Luz (2000) iniciou uma série de estudos sobre as espécies do gênero Panacris, examinando os tipos das duas espécies relacionadas; considerou $P$. proxima Kertész, 1908 sinônimo júnior de P. lucida. Posteriormente, Pujol-Luz \& Assis-Pujol (2002) revalidaram o gênero monotípico Spyridopa Gerstacker, 1857 (espécie-tipo Spyridopa tarsalis Gerstacker, 1857; anteriormente Panacris tarsalis) com base no exame do holótipo. Em seguida, Pujol-Luz \& Galinkin (2004) propuseram o gênero monotípico Tijucameru para Panacris maxima Kertész, 1908.

No presente trabalho, P. microdonta é transferida para o novo gênero Iidemyia, com base em estudos comparativos da terminália do macho, destacando a forma do hipândrio, dos gonocoxitos, dos gonóstilos e do edeago. A identificação dessa espécie foi realizada de acordo com as características descritas por James et al. (1980) e tomou por base a forma e posição dos espinhos escutelares, que diferem dos padrões conhecidos e descritos para as outras espécies dos gêneros afins de acordo com James et al. (1980), Pujol-Luz (2000), PujolLuz \& Assis-Pujol (2002) e Pujol-Luz \& Galinkin (2004). Os síntipos depositados na Zoologische Staatssammlung, 
Alemanha, não foram examinados porque os exemplares disponíveis para este trabalho combinam perfeitamente com as figuras e descrições anteriores para esta espécie encontradas principalmente na descrição original de Kertész (1908) e em James et al. (1980).

A terminologia adotada nas descrições foi adaptada de McAlpine (1981) e Pujol-Luz (2000). Os acrônimos das instituições citadas no texto são: HNHM, Magyar Természettudományi Múzeum, Budapest, Hungria; MZSP, Museu de Zoologia da Universidade de São Paulo, São Paulo; ZSBS, Zoologische Staatssammlung, Munique, Alemanha.

\section{Iidemyia, gen. nov.}

Espécie-Tipo: Panacris microdonta Kertész, 1908: 366.

Etimologia: Homenagem ao entomologista brasileiro, professor e amigo, Dr. Paulo Iide.

Diagnose: Coloração geral azul-escura a preta com discretos reflexos metálicos. Cabeça semi-esférica com largura máxima inferior à maior distância entre os calos umerais em ambos os sexos. Machos holópticos e fêmeas dicópticas; olhos compostos com pilosidade densa. Margens do occipício pouco desenvolvidas. Gena suavemente projetada para baixo, com pilosidade clara e esparsa. Triângulo ocelar pequeno e protuberante. Fronte e face suavemente convexas. Antena com flagelo globular no macho e com a extremidade mais afilada na fêmea; arista longa, proporcionalmente mais desenvolvida no macho. Disco do tórax azulado a preto brilhante, com pilosidade preta, curta e densa. Mesotórax com um calo pós-sutural supraalar em forma de concha. Escutelo convexo, com as margens laterais encurvadas, convergindo em direção à margem posterior que é semi-circular em vista dorsal, preto brilhante a azulado, com pilosidade esparsa e preta. Espinhos escutelares muito curtos e robustos localizados lateralmente no limite do terço posterior do escutelo, no início da margem posterior. Halteres amarelados. Tarsômeros castanho-escuros.

\section{Iidemyia microdonta (Kertész, 1908), comb. nov.}

Panacris microdonta Kertész, 1908: 366. Síntipo (macho e fêmea) HNHM, destruídos: Peru, Huánuco, foz do rio Pachitea; Síntipos (macho e fêmea) HNHM, destruídos: Peru, Ucayali, Mishagua [como Meshagua, erro], rio Urubamba; Síntipos (macho e fêmea) HNHM, destruídos: México; Síntipos (macho e fêmea) ZSBS: Costa Rica. (segundo Woodley, 2001: 127).

Registro da distribuição geográfica: Neotropical: Bolívia; Brasil (novo registro) (Espírito Santo e São Paulo); Costa Rica; El Salvador; Guatemala; México (Chiapas, Nayarit, Oaxaca, San Luís Potosí, Sinaloa, Veracruz); Panamá; Peru; Venezuela.

Macho. Cabeça semi-esférica, castanho-escura, largura máxima não maior que a distância entre os extremos dos calos umerais (Fig. 1). Triângulo ocelar protuberante (Figs. 2-3). Olhos compostos holópticos (Figs. 3), com pilosidade curta e densa. Occipício castanho escuro, relativamente estreito ao longo das margens oculares (Fig. 2). Fronte e face pouco protuberantes, suavemente convexas em vista lateral e

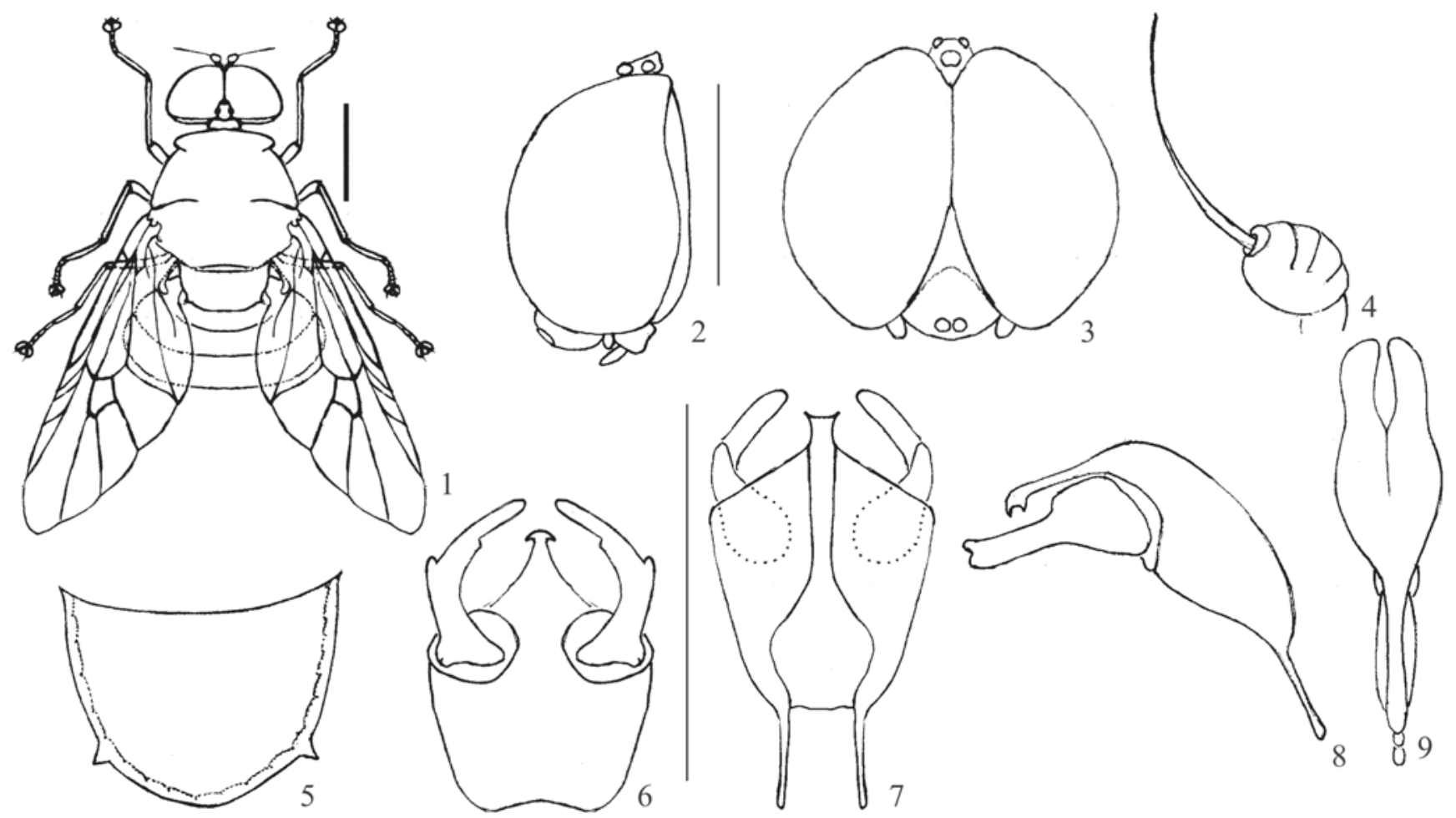

Figs. 1-9. Iidemyia microdonta. (Kertész, 1908), comb. nov. 1, Habitus do macho (vista dorsal); 2, Cabeça (vista lateral); 3, Cabeça (vista frontal); 4, Antena; 5, Escutelo (vista dorsal); 6, Cápsula genital (vista ventral); 7, Cápsula genital (vista dorsal); 8, Cápsula genital (vista lateral); 9, Edeágo (vista dorsal). 

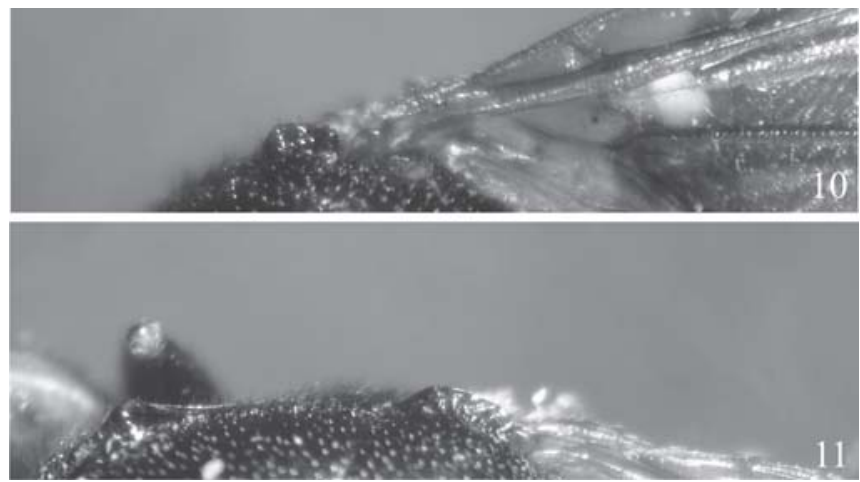

Figs. 10-11. Iidemyia microdonta. (Kertész, 1908), comb. n. 10, Calo pós-sutural supra-alar do macho (vista dorsal); 11, Calo pós-sutural supra-alar da fêmea (vista lateral).

delimitadas dos olhos por estreitas faixas de pilosidade prateada (Fig. 2). Antenas castanho-claras, com escapo e pedicelo caliciformes; flagelo globular (Fig. 4), flagelômeros muito curtos e parcialmente fusionados entre si e revestidos de pilosidade esparsa; arista mais longa que o complexo antenal, de coloração castanho-escura, com diminutas cerdas esparsas na base. Tórax preto-brilhante a azul escuro com reflexos metálicos discretos, revestido de pilosidade curta e esparsa (Fig. 10). Mesotórax com os calos pós-suturais supraalares pequenos, cerca da metade do comprimento do da fêmea, em forma de concha, com a margem crenulada (Fig. 10). Escutelo convexo, de cor semelhante ao restante do mesonoto, margens laterais encurvadas, convergindo em direção à margem do terço posterior, onde estão dois espinhos divergentes, robustos e muito curtos, com a metade distal castanho-escura ou avermelhada, delimitando a margem posterior em forma de arco de círculo (Figs. 5, 10). Asas hialinas com uma mácula castanho-escura limitada pelas células bm, br e dm e veias R1 e R2+3, também escurecidas; venação característica com br e bm bem diferenciadas, $r-m$ e cup presentes, célula dm pequena e trapezoidal, localizada na região mediana da asa; A1 fortemente retilínea; M1 suavemente curvada e M2 retilínea, conspícuas e alcançando a margem da asa (Fig. 1). Pernas pretas a castanho-escuras; sem esporões tibiais. Abdome arredondado e robusto, bem mais curto que o tórax, preto-brilhante a azul com reflexos metálicos, coberto com uma camada esparsa de pêlos claros. Terminália com epândrio semicircular; tergito $10 \mathrm{com}$ cercos digitiformes, gonocoxitos formando um conjunto subtrapezoidal com apódemas desenvolvidos e alongados; gonóstilos digitiformes e arqueados em direção à linha mediana, com a parte basal articulada localizada no terço distal dos gonocoxitos, estendendo-se além da extremidade distal do hipândrio, com um curto processo digitiforme no início da metade posterior da margem externa (Figs. 6-7); hipândrio sub-pentagonal, tendo a extremidade distal mais alongada e protuberante, com dois processos curtos e espiniformes (Fig. 7), que em vista lateral (Fig. 8) se assemelham a um par de ganchos curvos; edeago longo (Fig. 9), mais largo na metade distal e bífido, com os dois lobos projetados muito além da extremidade distal dos gonocoxitos; parâmeros não observados.

Comprimento total: $5 \mathrm{~mm}$, comprimento da asa: $6 \mathrm{~mm}$.

Fêmea. Semelhante ao macho, diferindo nos seguintes aspectos: olhos compostos dicópticos; occipício amarelado; fronte castanho-escura e pilosa, com faixas mais claras marginando os olhos, entre o triângulo ocelar e a face; face e gena com faixas laterais de pilosidade prateada, ausente na região mediana que é glabra; mesotórax com os calos póssuturais supra-alares duas vezes maiores que o do macho, em forma de concha, com a margem e a superfície crenuladas (Fig. 11); pernas castanho-escuras; cercos bissegmentados.

Comprimento total: $7,5 \mathrm{~mm}$, comprimento da asa: $7 \mathrm{~mm}$.

Material examinado: Dois machos e uma fêmea (MZSP): Brasil Espírito Santo, Córrrego do Itá, XI-XII.1956, W. Zikan col. (1 fêmea) São Paulo, Araçatuba, rio Jacarecatinga, X.1961, Lane \& Rabello col. (1 macho); Porto Albano, X.1954, E. Rabello col. (1 macho, abdome dissecado).

Agradecimentos. Aos colegas Nelson Papavero, Reginaldo Constantino e Cristiane Pujol, pela revisão do manuscrito. Ao Conselho Nacional de Desenvolvimento Científico e Tecnológico (Processo CNPq 308636/2007-4 e 474081/2007-9).

\section{REFERÊNCIAS}

Gerstaecker, A. 1857. Beitrag zur Kenntniss exotischer Stratiomyiden. Linnaea entomologica 11: 261-350.

James, M. T. 1967. A preliminary review of the Argentine genera and species of Stratiomyidae (Diptera). Part 2. Pachygasterinae. Acta zoologica lilloana 21: 95-121.

James, M. T.; M. W. McFadden \& N. E. Woodley. 1980. The Pachygastrinae (Diptera, Stratiomyidae) of Middle America. Melanderia 34: 1-36.

Kertész, K. 1908. Vorarbeiten zu einer Monographie der Notacanthen. XII-XXII. Annales Musei nationalis hungarici 7: 369-397.

Lindner, E. 1964. Beitrag zur Kenntnis der neotropischen Pachygasterinae (Stratiomyidae, Dipt.). Stuttgarter Beiträge zur Naturkunde 129: 1-22.

McAlpine, J. F. 1981. Morphology and terminology - adults, p. 9-63. In: McAlpine, J. F., Peterson, B. V., Shewell, G. E., Teskey, H. J., Vockeroth, J. R. \& D. M. Wood. (Eds.), Manual of Neartic Diptera. Volume 1. Monograph $\mathrm{N}^{\circ}$. 27. Research Branch, Agriculture Canada, Otawa, iv+674 p.

Pujol-Luz. J. R. 2000. Panacris proxima Kertész, 1908, new synonym of Panacris lucida Gerstaecker, 1857 (Diptera, Stratiomyidae) with notes on the male terminalia. Studia dipterologica 7: 155159.

Pujol-Luz. J. R. \& C. V. Assis-Pujol. 2002. Revalidação de Spyridopa Gerstaecker, 1857 (Diptera, Stratiomyidae). Boletim do Museu Nacional (NS), Zoologia, Rio de Janeiro 491: 1-5.

Pujol-Luz, J. R. \& J. Galinkin. 2004. Um novo gênero de Pachygastrinae (Diptera, Stratiomyidae) do Brasil. Neotropical Entomology 31: $35-38$.

Woodley, N. E. 2001. A World Catalog of the Stratiomyidae (Insecta, Diptera). Myia 11: 1-473. 